\title{
A halo approach to the evaluation of the cross-correlation between the SZ sky and galaxy surveys
}

\author{
Y. Qu and X.-P. Wu
}

\author{
National Astronomical Observatories, Chinese Academy of Sciences, Beijing 100012, PR China \\ e-mail: quyan@class2.bao.ac.cn
}

Received 17 February 2003 / Accepted 19 February 2004

\begin{abstract}
Using a purely analytic approach to gaseous and dark matter halos, we study the cross-correlation between the Sunyaev-Zel'dovich (SZ) sky and galaxy surveys under flat sky approximation, in an attempt to acquire the redshift information of the SZ map. The problem can be greatly simplified when it is noticed that the signals of the SZ-galaxy correlation arise only from hot gas and galaxies inside the same massive halos (i.e. clusters), and that field galaxies make almost no contribution to the cross-correlation. Under the assumption that both the hot gas and galaxies trace the common gravitational potential of dark halos, we calculate the expected cross SZ-galaxy power spectra for the WMAP/Planck SZ maps and the SDSS galaxy sample at small scales $100<l<1000$. It turns out, however, that it is not presently feasible to measure such small angular cross power spectra because of the high noise levels at $l>400$ in the WMAP/Planck experiments. Future SZ observations with better angular resolutions and sufficiently wide sky coverage will be needed if this technique is to be applied for the statistical measurement of the redshift distribution of the SZ sources.
\end{abstract}

Key words. cosmology: theory - cosmology: cosmic microwave background - cosmology: large-scale structure of Universe

\section{Introduction}

Most of the baryons in the local universe exist in the form of warm-hot intergalactic medium with temperature of $T \sim$ $10^{5}-10^{7} \mathrm{~K}$ as a result of gravitationally driven shocks and adiabatical compression when they fall into large-scale structures and collapsed dark matter halos (e.g., Cen \& Ostriker 1999; Davé et al. 2001). In the latter case, the very hot baryons gravitationally bound in massive halos such as groups and clusters usually manifest themselves as strong diffuse X-ray sources of bremsstrahlung emission, which are directly detectable with current X-ray instruments. Moreover, the energetic electrons of the hot gas also interact with the passing cosmic microwave background (CMB) photons through the so-called SunyaevZel'dovich (SZ) effect, giving rise to a subtle change in the CMB spectrum. Current SZ measurements of known clusters at high signal-to-noise are now routine (e.g., Carlstrom et al. 2002) and recent detection of the excess power relative to the primordial CMB anisotropy on arcminute scales has been successfully attributed to the statistical signals of the thermal SZ effect (Mason et al. 2003; Bond et al. 2003; Komatsu \& Seljak 2002).

However, both X-ray and SZ measurements contain no information about the redshift distributions of the hot baryons and their host groups/clusters. Spectroscopic follow-up observations should be made to complement our knowledge of the location and evolution of the hot baryons and their host halos. It has been realized that a more practical and powerful approach to extracting the distance information from the X-ray and SZ maps is perhaps to cross-correlate the X-ray and SZ maps with the existing galaxy catalogs or ongoing deep galaxy surveys (e.g., Seljak et al. 2000; Zhang \& Pen 2001, 2003; Zhang et al. 2002). In particular, the problem can be greatly simplified if we notice that the signals of the cross-correlation between the SZ (or X-ray) map and galaxy survey arise primarily from cluster galaxies, and that field galaxies make almost no contribution to the SZ- (or X-ray)-galaxy correlation. This permits a straightforward calculation of the power spectrum of the SZ- (or X-ray)-galaxy correlation from an analytic model of dark halo abundance, i.e., the Press \& Schechter (1974, PS) formalism, along with a reasonable prescription of galaxy and gas distributions inside a given halo of mass $M$ at redshift $z$. For the latter, one can adopt either the simplest self-similar NFW-like profile suggested by numerical simulations (Navarro et al. 1995, NFW), or the empirically motivated density profiles such as the King model or $\beta$-model, in combination with the halo occupation distribution (see Cooray \& Sheth 2003, for a recent review). In this paper, we explore the power spectrum of the SZ-galaxy cross-correlation based purely on the halo approach. A similar technique has been recently applied by Zhang \& Pen (2003) to the study of the cross-correlation of the soft X-ray background and galaxy surveys and by Wu \& Xue (2003) to the study of the auto-correlation of the soft X-ray background. In their earlier work, Zhang \& Pen (2001) also investigated the SZ-galaxy cross-correlation using the so-called continuum field model, in which the gas distribution in dark 
halos is given by a convolution of the dark matter distribution with a Gaussian window function, while the matter fluctuation in the highly non-linear regime (e.g. clusters) is calculated in terms of the hyper-extended perturbation theory.

For the purpose of actual applications, we will calculate the expected cross-correlation between the SZ maps observed by WMAP/Planck and the galaxy survey by the Sloan Digital Sky Survey (SDSS), and assess the feasibility of extracting the redshift information of the WMAP/Planck SZ maps using this statistical approach. Throughout this paper we adopt a flat cosmological model $(\Lambda \mathrm{CDM})$ with the best fit parameters determined by WMAP: $\Omega_{\Lambda}=0.73, \Omega_{\mathrm{M}}=0.27, h=0.71, \Omega_{\mathrm{b}} h^{2}=0.0224$, $\sigma_{8}=0.84$ and $n_{\mathrm{s}}=0.93$.

\section{SZ-galaxy cross-correlation}

\subsection{Properties of gaseous and dark matter halos}

The thermal SZ effect along the direction $\boldsymbol{\theta}$ due to the hot gas inside a halo can be evaluated following

$$
\begin{aligned}
\frac{\Delta T(\boldsymbol{\theta})}{T_{\mathrm{CMB}}} & =g(x) y(\boldsymbol{\theta}) ; \\
y(\boldsymbol{\theta}) & =\int N_{\mathrm{e}} \sigma_{T}\left(\frac{k_{\mathrm{B}} T}{m_{\mathrm{e}} c^{2}}\right) \mathrm{d} \chi ; \\
g(x) & =\frac{x^{2} \mathrm{e}^{x}}{\left(\mathrm{e}^{x}-1\right)^{2}}\left(4-x \operatorname{coth} \frac{x}{2}\right),
\end{aligned}
$$

where $N_{\mathrm{e}}$ and $T$ are the number density and temperature of electrons, $x=h_{\mathrm{p}} v / k_{\mathrm{B}} T_{\mathrm{CMB}}$ is the dimensionless frequency, $T_{\mathrm{CMB}}$ is the temperature of the present $\mathrm{CMB}$, and the integral is performed along the line of sight. We specify the gas temperature in terms of the virial theorem

$k_{\mathrm{B}} T=1.39 \mathrm{keV} f_{T}\left(\frac{M}{10^{15} M_{\odot}}\right)^{2 / 3}\left[h^{2} E^{2}(z) \Delta_{\mathrm{c}}\right]^{1 / 3}$,

where $\Delta_{\mathrm{c}}$ is the overdensity of dark matter with respect to the critical value $\rho_{\text {crit }}$, which is calculated using a fitting formula given by Bryan \& Norman (1998) for $f_{T}=0.8$, and $E^{2}(z)=$ $\Omega_{\mathrm{M}}(1+z)^{3}+\Omega_{\Lambda}$.

For simplicity we assume that both gas and galaxies in massive halos trace the dark matter distribution such that

$N_{\mathrm{e}}(r)=\frac{f_{\mathrm{b}}}{\mu_{\mathrm{e}} m_{\mathrm{p}}} \Delta \rho_{\mathrm{DM}}(r) ;$

$\Delta N_{\mathrm{gal}}(r)=A \Delta \rho_{\mathrm{DM}}(r)$,

in which $f_{\mathrm{b}}$ is the universal baryon fraction, $\mu_{\mathrm{e}}=1.13$ is the mean electron weight, $\Delta N_{\text {gal }}(r)$ is the number density of galaxies, and $A$ is a proportionality constant. Indeed, this so-called self-similar model provides a good approximation for the radial profiles of gas and galaxies in dark halos if our focus is not on the detailed physical processes of the gas and galaxies. Unlike thermal X-ray emission which is governed by the central gas content of massive dark halos such as groups and clusters of galaxies, the thermal SZ effect is generated by the hot gas distributed over whole halo regions. Beyond the core regions the radial profiles of gas and dark matter look very similar. In recent years some physically motivated models have been proposed for the density and temperature of the hot gas in massive halos (e.g., Babul et al. 2002; Voit et al. 2002; Wu $\&$ Xue 2002; etc.). One can easily extend our formalism to include these gas profiles. Here we adopt the universal density profile as suggested by NFW to describe the dark matter distribution inside halos, $\Delta \rho_{\mathrm{DM}}(r)=\delta_{\mathrm{ch}} \rho_{\text {crit }} /\left[\left(r / r_{\mathrm{s}}\right)\left(1+r / r_{\mathrm{s}}\right)^{2}\right]$, and fix the free parameter using the empirical fitting formula found by numerical simulations (Bullock et al. 2001): $c=$ $[9 /(1+z)]\left(M / 2.1 \times 10^{13} M_{\odot}\right)^{-0.13}$, where $c=r_{\text {vir }} / r_{\mathrm{s}}$ is the socalled concentration parameter.

To determine the constant $A$ in Eq. (6), we need to know the mean galaxy number for a given halo of mass $M$, i.e. the halo occupation distribution, $N_{\mathrm{gal}}(M)$. We use the best fit analytic formulae of Sheth \& Diaferio (2001) for the spiral $\left[N_{\mathrm{gal}, \mathrm{S}}(M)\right]$ and elliptical $\left[N_{\text {gal,E }}(M)\right]$ galaxies based on the GIF simulations (Kauffmannn et al. 1999):

$$
\begin{aligned}
N_{\mathrm{gal}, \mathrm{S}}(M) & =\left(M / M_{\mathrm{S}}\right)^{\alpha_{\mathrm{S}}}+0.5 \mathrm{e}^{-4\left[\log \left(M / 10^{11.75} M_{\odot}\right)\right]^{2}} \\
N_{\mathrm{gal}, \mathrm{E}}(M) & =\left(M / M_{\mathrm{E}}\right)^{\alpha_{\mathrm{E}}} \mathrm{e}^{-\left(2 \times 10^{11} M_{\odot} / M\right)^{2}} \\
N_{\mathrm{gal}}(M) & =N_{\mathrm{gal}, \mathrm{S}}(M)+N_{\mathrm{gal}, \mathrm{E}}(M)
\end{aligned}
$$

where $M_{\mathrm{S}}=7 \times 10^{13} h^{-1} M_{\odot}, \alpha_{\mathrm{S}}=0.9, M_{\mathrm{E}}=3 \times 10^{12} h^{-1} M_{\odot}$, and $\alpha_{\mathrm{E}}=0.75$. The mean number density of galaxies is thus

$\bar{N}_{\text {gal }}=\int N_{\text {gal }}(M) \frac{\mathrm{d}^{2} N}{\mathrm{~d} M \mathrm{~d} V} \mathrm{~d} M$,

where $\mathrm{d}^{2} N / \mathrm{d} M \mathrm{~d} V$ is the comoving number density of dark halos (see below). Given the halo occupation distribution, we are able to normalize the number density profile of galaxies within a halo of mass $M$ following

$N_{\text {gal }}(M)=\int \Delta N_{\text {gal }}(r) \mathrm{d} V$.

The integration above is made over the whole cluster region out to the virial radius. This gives the proportionality constant $A$ in Eq. (6)

$A=\frac{N_{\mathrm{gal}}(M)}{4 \pi \delta_{\mathrm{ch}} \rho_{\mathrm{crit}} r_{\mathrm{s}}^{3}[\ln (1+c)-c /(1+c)]}$.

Now, one can easily get the surface number density of galaxies by projecting $\Delta N_{\text {gal }}(r)$ along the line of sight $\boldsymbol{\theta}$ :

$n_{\mathrm{gal}}(M, z, \boldsymbol{\theta})=D_{\mathrm{A}}^{2}(z) \int \Delta N_{\mathrm{gal}}(r) \mathrm{d} \chi$,

in which $D_{\mathrm{A}}(z)$ is the angular diameter distance to the halo.

\subsection{Power spectra}

The angular cross power spectrum of the SZ-galaxy correlation can be separated into the Poisson term $C_{l}^{\mathrm{P}}$ and the clustering term $C_{l}^{\mathrm{C}}$ :

$$
\begin{aligned}
C_{l}^{\mathrm{P}}= & g(x) \int \mathrm{d} z \frac{\mathrm{d} V}{\mathrm{~d} z \mathrm{~d} \Omega} B\left(z, z_{0}\right) \\
& \times \int \mathrm{d} M \frac{\mathrm{d}^{2} N(M, z)}{\mathrm{d} M \mathrm{~d} V}\left|y_{l}(M, z) n_{l}(M, z)\right|,
\end{aligned}
$$


and

$$
\begin{aligned}
C_{l}^{\mathrm{C}}= & g(x) \int \mathrm{d} z \frac{\mathrm{d} V}{\mathrm{~d} z \mathrm{~d} \Omega} P\left(l / D_{0}, z\right) B\left(z, z_{0}\right) \\
& \times\left[\int \mathrm{d} M \frac{\mathrm{d}^{2} N(M, z)}{\mathrm{d} M \mathrm{~d} V} b(M, z) y_{l}(M, z)\right] \\
& \times\left[\int \mathrm{d} M \frac{\mathrm{d}^{2} N(M, z)}{\mathrm{d} M \mathrm{~d} V} b(M, z) n_{l}\right],
\end{aligned}
$$

where $D_{0}$ is the comoving angular diameter distance to the halo of mass $M$ at $z$, and $y_{l}$ and $n_{l}$ are the Fourier transforms of the Compton $y$-parameter and the surface overdensity of galaxies $\left[n_{\text {gal }}(M, z, \boldsymbol{\theta})-\bar{n}_{\text {gal }}\left(z_{0}\right)\right] / \bar{n}_{\text {gal }}\left(z_{0}\right)$, respectively, $b(M, z)$ is the bias parameter, for which we use the analytic prescription of Mo \& White (1996), and the mean surface number density of galaxies $\bar{n}_{\text {gal }}\left(z_{0}\right)$ is given by

$\bar{n}_{\text {gal }}\left(z_{0}\right)=\int \bar{N}_{\text {gal }}(z)(1+z)^{2} B\left(z, z_{0}\right) D_{\mathrm{A}}^{2}(z) \frac{\mathrm{d} \chi}{\mathrm{d} z} \mathrm{~d} z$,

where, as in Eqs. (12) and (13), $B\left(z, z_{0}\right)$ denotes the redshift distribution function of galaxies. Here we use the following approximate expression (e.g., Baugh \& Efstathiou 1993; Dodelson et al. 2002):

$B\left(z, z_{0}\right)=\frac{3 z^{2}}{2\left(z_{0} / 1.412\right)^{3}} \mathrm{e}^{-\left(1.412 z / z_{0}\right)^{3 / 2}}$,

in which $z_{0}$ is the median redshift of the galaxies in the galaxy survey.

Finally, we adopt the PS mass function for the comoving number density of dark halos

$$
\frac{\mathrm{d}^{2} N}{\mathrm{~d} M \mathrm{~d} V}=-\sqrt{\frac{2}{\pi}} \frac{\bar{\rho}}{M} \frac{\delta_{\mathrm{c}}(z)}{\sigma^{2}(M)} \frac{\mathrm{d} \sigma(M)}{\mathrm{d} M} \exp \left(-\frac{\delta_{\mathrm{c}}^{2}(z)}{2 \sigma^{2}(M)}\right),
$$

in which $\delta_{\mathrm{c}}$ is the linear over-density of perturbations that collapsed and virialized at redshift $z$, and $\sigma(M)$ is the linear theory variance of the mass density fluctuation in a sphere of mass $M$ :

$\sigma^{2}(M)=\frac{1}{2 \pi^{2}} \int_{0}^{\infty} k^{2} P(k)|W(k R)|^{2} \mathrm{~d} k$,

and $W(x)=3(\sin x-x \cos x) / x^{3}$ is the Fourier representation of the window function. The power spectrum, $P(k) \propto k^{n_{\mathrm{s}}} T^{2}(k)$, is normalized by the rms fluctuation on an $8 h^{-1} \mathrm{Mpc}$ scale, $\sigma_{8}$, and we take the transfer function $T(k)$ from an adiabatic CDM model given by Bardeen et al. (1986). If we replace $n_{l}$ by $g(x) y_{l}$ and set $B\left(z, z_{0}\right)=1$ in Eqs. (12) and (13) we can get the thermal SZ power spectrum, $C_{l}^{\mathrm{SZ}-\mathrm{SZ}}$. Instead, if $g(x) y_{l}$ is replaced by $n_{l}$, we will obtain the galaxy-galaxy correlation, $C_{l}^{\text {gal-gal }}$. Note that for the latter case the square of $\left|n_{l}\right|$ in Eq. (12) should be $\left|n_{l}\right|$ if $\left\langle N_{\text {gal }}\left(N_{\text {gal }}-1\right)\right\rangle<1$ (Seljak 2000).

\section{Application: Prediction for the cross-correlation between the MAP/Planck SZ maps and SDSS galaxy survey}

First, we demonstrate in Fig. 1 the expected power spectra of auto-correlations of the SZ sky and galaxy survey and their cross-correlation at frequency $v=30 \mathrm{GHz}$ and for all galaxies

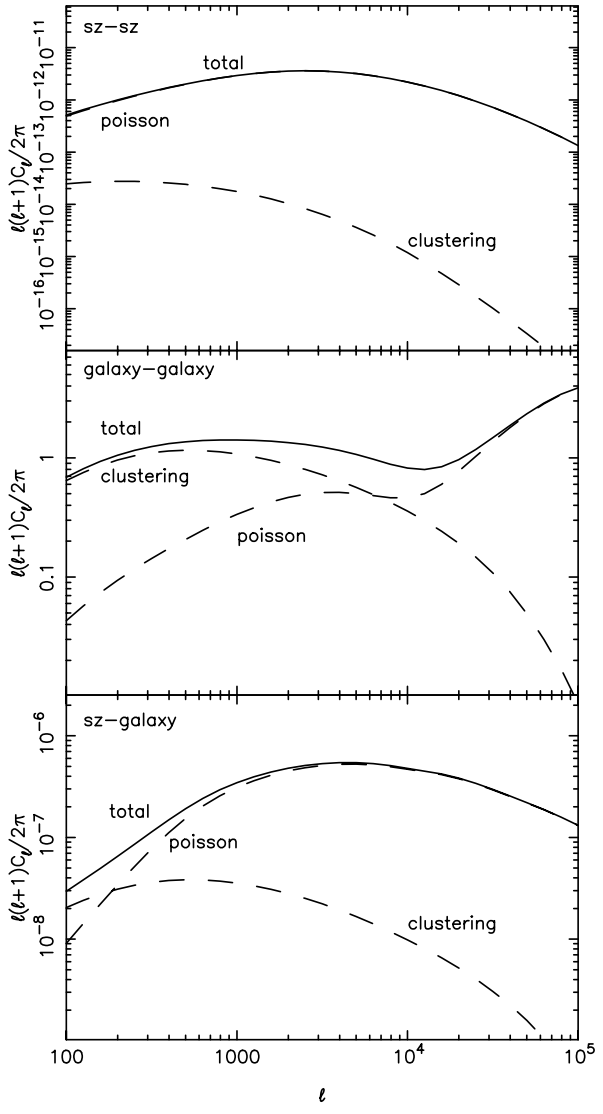

Fig. 1. The angular power spectra of the SZ-SZ (top panel), galaxygalaxy (middle panel) and SZ-galaxy (lower panel) correlations at frequency $v=30 \mathrm{GHz}$ and for all galaxies. Poisson and clustering components are explicitly displayed.

$(B=1)$. Note that we have dropped the negative sign in $g(x)$. Essentially, the SZ-SZ power spectrum is dominated by a Poisson distribution over the whole multipole range from $l=$ 100 (e.g., Komatsu \& Kitayama 1999), while clustering of the sources governs the galaxy-galaxy correlation at angular scales up to $l \approx 5000$. Overall, both the SZ-SZ and SZ-galaxy power spectra exhibit a decline at sufficiently large $l$, regardless of the fact that the galaxy-galaxy correlation still has much power at high $l$ beyond $l=10^{4}$. The latter can be attributed to pairs of galaxies residing within the same, smaller halos at higher redshifts which are included in the estimate of galaxy-galaxy correlation because of the choice $B=1$. Extrapolation of the halo occupation number to high redshifts may lead to an overestimate of galaxies in small halos. Note that unlike the Compton $y$-parameter, the galaxy surface number density $n_{\mathrm{gal}}(M, z, \boldsymbol{\theta})$ is proportional to $D_{\mathrm{A}}^{2}(z)$ though they have the same spatial dependence on the underlying dark matter distribution of clusters. The strong power of the galaxy-galaxy correlation at high $l$ can be significantly suppressed when we work with a realistic redshift distribution function $B\left(z, z_{0}\right)$ based on galaxy surveys such as SDSS (see below).

Next, following the investigation of Peiris \& Spergel (2000), we carry out our numerical predictions for the galaxy sample of SDSS and the SZ sky (to be) revealed by WMAP/Planck surveyor. We study the feasibility of extracting the redshift information of the SZ sky from their angular 
cross power spectra. To this end we select the SDSS galaxies in different magnitude ranges characterized by different values of median redshift $z_{0}$ in the distribution function $B\left(z, z_{0}\right)$ (Dodelson et al. 2002). We assume that all the galaxies have photometrically determined redshifts to optimize the utilization of the SDSS data. This yields a total of $1.2 \times 10^{8}$ galaxies over a quarter of the sky in the magnitude bin $20<r^{*}<21$. We have also compared the angular power spectra of galaxies $C_{l}^{\text {gal-gal }}$ predicted by our halo model with those determined by Tegmark et al. (2002) from early SDSS data on scales $l<600$ in four different magnitude ranges from $r^{*}=18$ to 22 , and found that the agreement is good when the bias factors suggested by Tegmark et al. (2002) are used in different magnitude bins (see Fig. 2 of Tegmark et al. 2002).

The working frequencies of both WMAP and Planck are fixed at $v \approx 30 \mathrm{GHz}$. Whether or not one is able to effectively measure the SZ-SZ, galaxy-galaxy and SZ-galaxy power spectra depends critically on the noise levels. Following the standard treatment, we estimate the errors in the SZ$\mathrm{SZ}$ power spectrum, $C_{l}^{\mathrm{SZ}-\mathrm{SZ}}$, and galaxy-galaxy power spectrum, $C_{l}^{\text {gal-gal }}$, through

$$
\begin{aligned}
\Delta C_{l}^{\mathrm{SZ}-\mathrm{SZ}} & =\frac{\sqrt{2}}{\sqrt{(2 l+1) \Delta l f_{\mathrm{sky}}^{\mathrm{SZ}}}} C_{l}^{\mathrm{SZ}-\mathrm{SZ}, \mathrm{tot}} ; \\
\Delta C_{l}^{\mathrm{gal}-\mathrm{gal}} & =\frac{\sqrt{2}}{\sqrt{(2 l+1) \Delta l f_{\mathrm{sky}}^{\mathrm{gal}}}} C_{l}^{\text {gal-gal,tot }},
\end{aligned}
$$

where $f_{\text {sky }}^{\text {SZ }}$ and $f_{\text {sky }}^{\text {gal }}$ are the MAP/Planck and SDSS sky coverages, respectively, and $\Delta l$ is the bin width that will be fixed to be $l / 4$ in our estimation (see Zhang et al. 2002). $C_{l}^{\text {tot }}$ denotes all contributions to the measured power spectra that are essentially composed of true signals and detector noise, $C_{l}^{\text {tot }}=$ $C_{l}^{\text {true }}+C_{l}^{\text {noise }}$, provided that foreground contamination is properly removed. For the SDSS survey, the shot noise per mode is simply

$$
C_{l}^{\text {noise }}=\frac{1}{n_{\text {gal }}} \text {. }
$$

For the SZ sky observed by MAP/Planck, the instrumental noise per mode can be modeled by

$C_{l}^{\text {noise }}=w^{-1}(v) \mathrm{e}^{\theta^{2}(v) l(l+1)}$,

in which we have assumed that the experimental beam is Gaussian with width $\theta^{2}(v)\left(F W H M=(8 \ln 2)^{1 / 2} \theta(v)\right)$, and $w^{-1 / 2}$ denotes the noise level in CMB temperature fluctuation per pixel. For our working frequency of $v \approx 30 \mathrm{GHz}$, we choose these parameters in terms of the tabulated values given by Cooray \& $\mathrm{Hu}(2000)$. In a similar way, we can estimate the error for the SZ-galaxy power spectrum from

$$
\begin{aligned}
\Delta C_{l}^{\mathrm{SZ}-\text { gal }}= & \frac{\sqrt{2}}{\sqrt{(2 l+1) \Delta l f_{\text {sky }}}}\left[\left(C_{l}^{\mathrm{SZ}-\text { gal }}\right)^{2}\right. \\
& \left.+C_{l}^{\mathrm{SZ}-\mathrm{SZ}, \text { tot }} C_{l}^{\text {gal-gal }, \text { tot }}\right]^{1 / 2} .
\end{aligned}
$$

We show in Fig. 2 the expected power spectra of the auto-correlations of the WMAP/Planck SZ maps and the

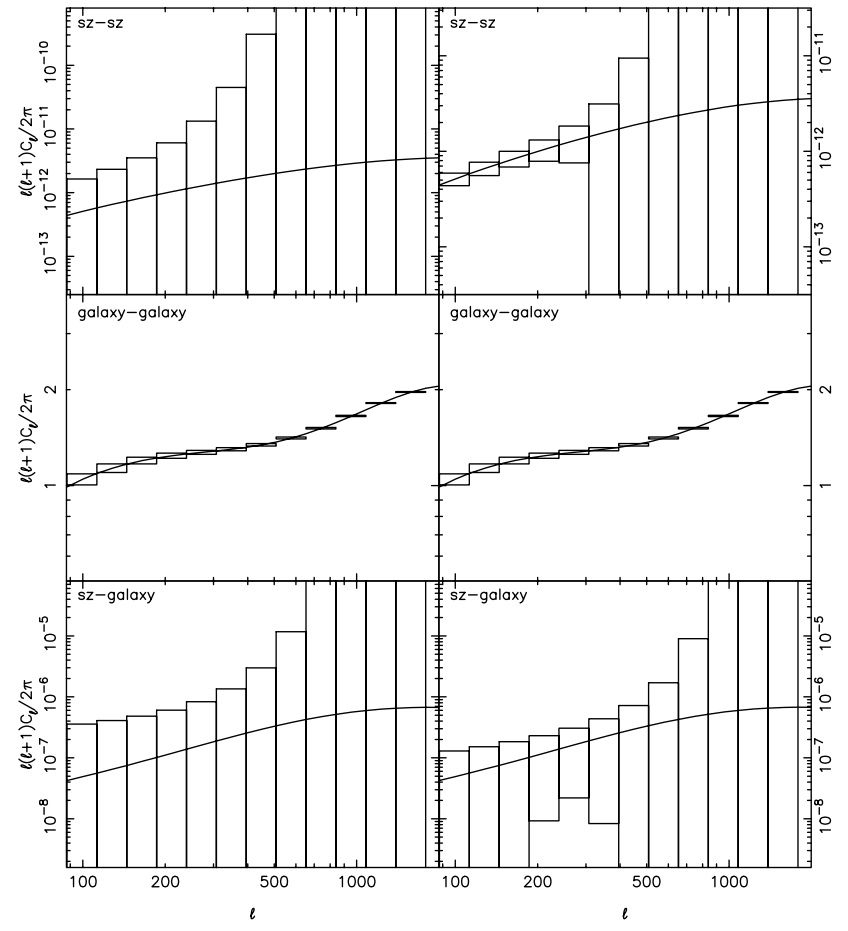

Fig. 2. Expected angular power spectra of the SZ-SZ (top panel), the SDSS galaxy-galaxy (middle panel) and the SZ-SDSS galaxy (lower panel) correlations at frequency $v=30 \mathrm{GHz}$ and for $z_{0}=0.33$. Bin width is chosen as $\Delta l=l / 4$. Left and right panels correspond to the WMAP and Planck results, respectively.

SDSS galaxy survey and their cross-correlations for $z_{0}=0.33$, corresponding to $20<r^{*}<21$. While in principle the galaxy power spectrum can be well constructed with the SDSS survey over a broad range of multipoles, the measurements of the SZ power spectra beyond $l \sim$ a few hundreds are hampered by the poor angular resolutions of the WMAP/Planck experiments, although the result of Planck is slightly better (see Bennett et al. (2003) for the first year WMAP result). Recall that in the observed CMB spectrum the SZ signals appear to be dominant only at $>2000$ unless the primary CMB can be accurately removed. Consequently, it may turn out to be impossible to measure the cross-correlation between the WMAP/Planck SZ sky and the SDSS galaxy survey. Yet, another possibility is to search for the cross-correlation signals at very large angular scales generated by superclusters (Hernández-Monteagudo \& Rubinõ-Martín 2003) or large-scale matter inhomogeneities at low redshifts (e.g. $z<0.1$ ) (Afshordi et al. 2004). Indeed, the noise appears to be relatively smaller at low redshifts and large angular scales (see Fig. 3), which facilitates the detection of the cross-correlation of the WMAP/Planck SZ maps with galaxy surveys such as SDSS and 2MASS, although in this case our primary goal of extracting redshift information of the SZ map from the cross-correlation signals becomes less interesting.

Taking the Planck experiment as an example, we have shown in Fig. 3 the SZ-galaxy cross-correlations for a set of median redshifts of the SDSS galaxies. Despite the large errors at high $l$ beyond $\sim 400$, it demonstrates the actual procedures that one needs to perform in order to extract the redshift information of the SZ map from these cross-correlations. 


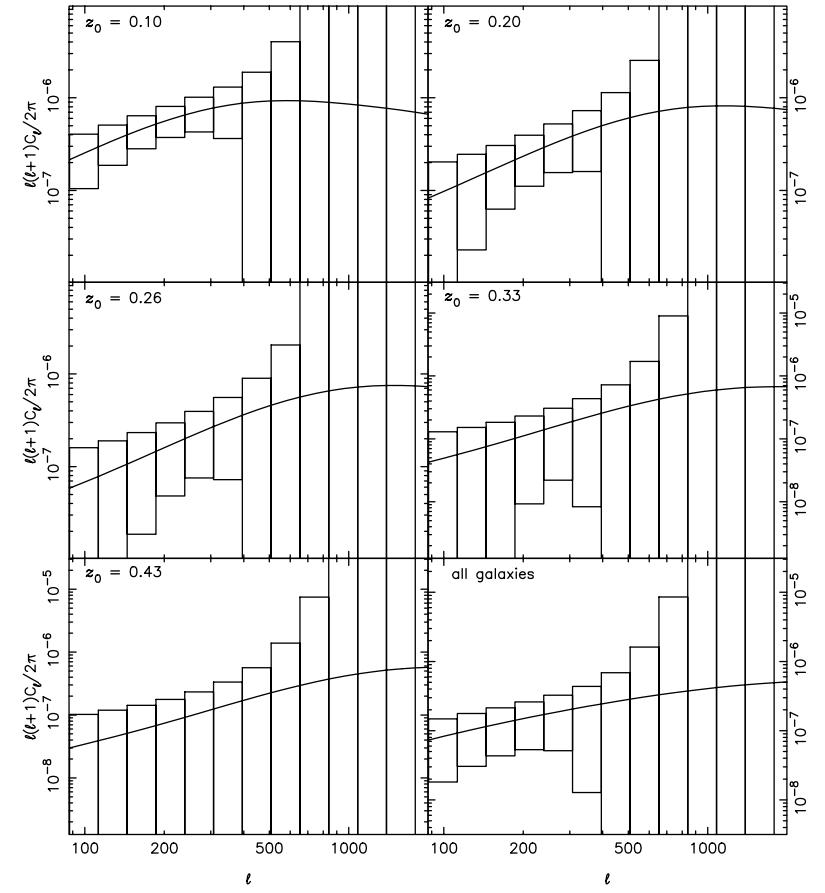

Fig. 3. Dependence of the Planck SZ-galaxy power spectra on redshifts of the SDSS galaxies.

Finally, we calculate the correlation coefficient between the SZ map and the galaxy survey following

$r_{\text {corr }}(l)=\frac{C_{l}^{\mathrm{SZ}-\mathrm{gal}}}{\sqrt{C_{l}^{\mathrm{SZ}-\mathrm{SZ} C_{l}^{\mathrm{gal}-\mathrm{gal}}}}}$.

Recall that $r_{\text {corr }}(l)$ acts as a quantitative indicator of the significance of the SZ-galaxy correlation. The associated error can be estimated by

$$
\begin{aligned}
& (\Delta r)^{2}=r_{\text {corr }}^{2}(l)\left\{\left(\frac{\Delta C_{l}^{\mathrm{SZ}-\mathrm{gal}}}{C_{l}^{\mathrm{SZ}-\mathrm{gal}}}\right)^{2}+\frac{1}{2(2 l+1) f_{\mathrm{sky}} \Delta l}\right. \\
& \times\left(\frac{C_{l}^{\mathrm{SZ}-\mathrm{SZ}, \mathrm{tot}}}{C_{l}^{\mathrm{SZ}-\mathrm{SZ}}}+\frac{C_{l}^{\mathrm{gal}-\mathrm{gal}, \mathrm{tot}}}{C_{l}^{\mathrm{gal}-\mathrm{gal}}}\right) \\
& \times\left[\frac{C_{l}^{\mathrm{SZ}-\mathrm{SZ}, \mathrm{tot}}}{C_{l}^{\mathrm{SZ}-\mathrm{SZ}}}+\frac{C_{l}^{\mathrm{gal}-\mathrm{gal}, \mathrm{tot}}}{C_{l}^{\mathrm{gal}-\mathrm{gal}}}\right. \\
& \left.\left.-4\left(1+\frac{C_{l}^{\mathrm{SZ}-\mathrm{SZ}, \text { tot }} C_{l}^{\mathrm{gal}-\mathrm{gal}, \mathrm{tot}}}{\left(C_{l}^{\mathrm{SZ}-\mathrm{gal}}\right)^{2}}\right)^{1 / 2}\right]\right\} \text {. }
\end{aligned}
$$

We display in Fig. 4 the resulting correlation coefficients in the same redshift ranges as in Fig. 3 for the Planck SZ-SDSS galaxy correlation. Similarly to the power spectra in Fig. 3, instrumental noise becomes dominant at both lower and higher $l$ for the correlation coefficients. The peak location of $r_{\text {corr }}$ increases with the median redshift of SDSS galaxy distribution because inclusion of more distant galaxies in the estimate of the SZ-galaxy correlation raises the power at smaller angular scales. Overall, the correlation coefficient has a value of $r_{\text {corr }} \approx$ 0.2 at $100<l<1000$, indicating that the two phenomena are only moderately correlated. This arises from the fact that only

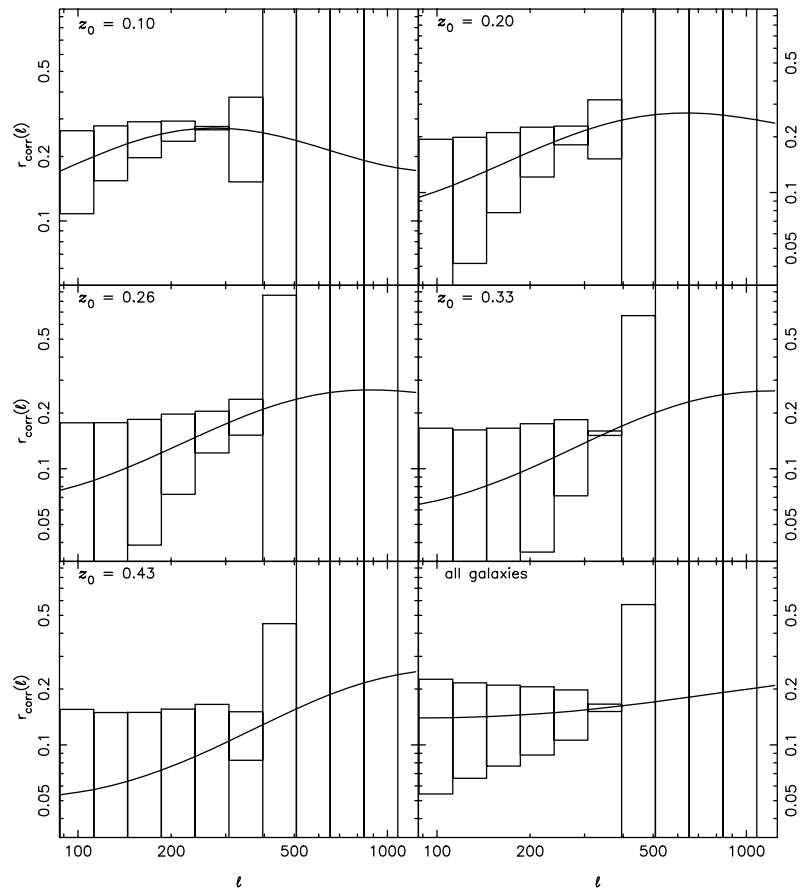

Fig. 4. The correlation coefficients for the Planck SZ map and SDSS galaxy survey in different redshift ranges. Bin width is taken to be $\Delta l=l / 4$.

galaxies in clusters can contribute to the SZ-galaxy correlation and field galaxies selected in SDSS sample have almost no effect on the cross correlation between the SZ map and the galaxy survey. Zhang \& Pen (2001) made a similar analysis to maximize $r_{\text {corr }}$ by properly picking the redshift distribution function of the SDSS galaxies, which can increase the correlation coefficient to $r_{\text {corr }}^{\max } \approx 0.7$ at a smaller scale of $10^{3}<l<10^{4}$.

\section{Discussion and conclusions}

Cross-correlation between the SZ map and galaxy surveys will reveal, in a statistical manner, important information on the redshift distribution of hot baryons in the universe, which requires no spectroscopic follow-up observation of individual sources. Recall that the major advantage of measurement of the SZ power spectrum over the SZ cluster survey is that one can acquire weak SZ signals at high statistical significance level without the need to resolve individual clusters. Indeed, time-consuming spectroscopic follow-up observations of the SZ sources may eventually throw a shadow on the effective applications of the SZ power spectrum where the redshift distribution is ultimately concerned. Cross-correlation between the SZ map and galaxy surveys provides a simple approach which is just suited for the problem. In particular, theoretical prediction of the SZ-galaxy power spectrum can be greatly simplified if one notices that the signals of the SZ-galaxy crosscorrelation are primarily produced by the hot gas and galaxies within the same clusters. Namely, field galaxies make almost no contribution to the SZ-galaxy cross-correlation.

Assuming that both the intracluster gas and cluster galaxies have the same dark matter distribution with a functional form of an NFW-like profile, and that the abundance of dark halos 
is described by the PS mass function, we have predicted the SZ-galaxy angular power spectrum. As is expected, the power spectrum indeed shows a moderately strong correlation indicated by an overall correlation coefficient of $r_{\text {corr }} \approx 0.2$ at $100<$ $l<1000$. However, applying our algorithm to the SDSS galaxy survey and MAP/Planck SZ maps yields an unpleasant result: it is unlikely that one can acquire meaningful information about the redshift distribution from the MAP/Planck SZ maps because the SZ signals are significantly below the noise levels in the range of our interested multipoles, $l>1000$, although the SDSS galaxy sample should be just suited for such a purpose. One possibility is to work at large angular scales $l<100$, which is related to the SZ effect produced by nearby superclusters and clustering of galaxies (Hernández-Monteagudo \& Rubinõ-Martín 2003; Afshordi et al. 2004). Another application is to utilize the CMB data at very small angular scales beyond $l=2000$ to be obtained by many upcoming experiments such as ACBAR, AMiBA, BIMA, CBI, etc. In this case, one needs instead to deal with the small-sky-coverage problem. Indeed, a similar study should be done on the feasibility of cross-correlating these coverage CMB data with existing galaxy catalogs. We conclude that the cross-correlation between the SZ map and galaxy surveys can in principle yield valuable information about the redshift distribution of the host baryons in the universe. However, actual application of this technique to real observations may not be possible until an SZ power spectrum with high angular resolution, high sensitivity and wide sky coverage is achieved.

Acknowledgements. Constructive suggestions by an anonymous referee are gratefully acknowledged. This work was supported by the National Science Foundation of China, under Grant No. 10233040, and the Ministry of Science and Technology of China, under Grant No. NKBRSF G19990754.

\section{References}

Afshordi, N., Loh, Y.-S., \& Strauss, M. A. 2004, Phys. Rev. D, 69, 083524
Babul, A., Balogh, M. L., Lewis, G. F., \& Poole, G. B. 2002, MNRAS, 330, 329

Bardeen, J. M., Bond, J. R., Kaiser, N., \& Szalay, A. S. 1986, ApJ, 304, 15

Baugh, C. M., \& Efstathiou, G. 1993, MNRAS, 265, 145

Bennett, C. L., Halpern, M., Hinshaw, G., et al. 2003, ApJS, 148, 1,

Bryan, G. L., \& Norman, M. L. 1998, ApJ, 495, 80

Bullock, J. S., Kolatt, T. S., Sigad, Y., et al. 2001, MNRAS, 321, 559

Bond, J. R., Contaldi, C. R., Pen, U.-L., et al. 2003, ApJ, in press [arXiv: astro-ph/0205386]

Carlstrom, J. E. 2002, ARA\&A, 40, 643

Cen, R., \& Ostriker, J. P. 1999, ApJ, 514, 1

Cooray, A., \& Hu, W. 2000, ApJ, 534, 533

Cooray, A., \& Sheth, R. 2003, Phys. Rep., 372, 1

Davé, R., Cen, R., Ostriker, J. P., et al. 2001, ApJ, 552, 473

Dodelson, S., Narayanan, V. K., Tegmark, M., et al. 2002, ApJ, 572, 140

Hernández-Monteagudo, C., \& Rubiño-Martín, J. A. 2004, MNRAS, 347,403

Kauffmann, G., Colberg, J. M., Diaferio, A., \& White, S. D. M. 1999, MNRAS, 303, 188

Komatsu, E., \& Kitayama, T. 1999, ApJ, 526, L1

Komatsu, E., \& Seljak, U. 2002, MNRAS, 336, 1256

Mason, B. S., Pearson, T. J., Readhead, A. C. S., et al. 2003, ApJ, 591, 540

Mo, H.-J., \& White, S. D. M. 1996, MNRAS, 282, 347

Navarro, J. F., Frenk, C. S., \& White, S. D. M. 1995, MNRAS, 275, 720

Peiris, H. V., \& Spergel, D. N. 2000, ApJ, 540, 605

Press, W. H., \& Schechter, P. 1974, ApJ, 187, 425

Seljak, U. 2000, MNRAS, 318, 203

Seljak, U., Burwell, J., \& Pen, U.-L. 2000, Phys. Rev. D, 63, 063001

Sheth, R. K., \& Diaferio, A. 2001, MNRAS, 322, 901

Tegmark, M. , Dedelson, S., Eisenstein, D. J., et al. 2002, ApJ, 571, 191

Voit, G. M., Bryan, G. L., Balogh, M. L., \& Bower, R. G. 2002, ApJ, 576,601

Wu, X.-P., \& Xue, Y.-J. 2002, ApJ, 569, 112

Wu, X.-P., \& Xue, Y.-J. 2003, ApJ, 590, 8

Zhang, P.-J., \& Pen, U.-L. 2001, ApJ, 549, 18

Zhang, P.-J., \& Pen, U.-L. 2003, ApJ, 588, 704

Zhang, P.-J., Pen, U.-L., \& Wang, B. 2002, ApJ, 577, 568 